\title{
Highly efficient gold nano-flowered optical biosensor doped in sol- gel/PEG matrix for the determination of calcitonin biomarker in different serum samples
}

\author{
Walaa. E. Omer ${ }^{1}$, Maged A. El-Kemary ${ }^{1}$, Mostafa M. Elsaady ${ }^{2}$, Mona N. Abou- \\ Omar $^{3}$, Ahmed O. Youssef ${ }^{2}$, Ali A. Sayqal ${ }^{4}$, Ayman A. Gouda ${ }^{5}$, Mohamed S. Attia ${ }^{2 *}$ \\ ${ }^{1}$ Institute of Nanoscience and Nanotechnology, Kafrelsheikh University, 33516 Egypt. ${ }^{2}$ Department of \\ Chemistry, Faculty of Science, Ain Shams University, 11566, Cairo, Egypt. \\ ${ }^{3}$ Department of Chemistry, Faculty of Women for Arts, Science and Education, Ain Shams University, Cairo, \\ Egypt. \\ ${ }^{4}$ Chemistry Department, Faculty of Applied Sciences, Umm Al-Qura University, Makkah, Saudi Arabia. \\ ${ }^{5}$ Faculty of Public Health and Health Informatics, Umm Al-Qura University, Makkah, Saudi Arabia. \\ Corresponding author: (M. S. Attia) \\ Mohamed_sam@yahoo.com, \\ mohd_mostafa@sci.asu.edu.eg, Tel: +(202)1229867311,+(202) 1060818922
}


S1

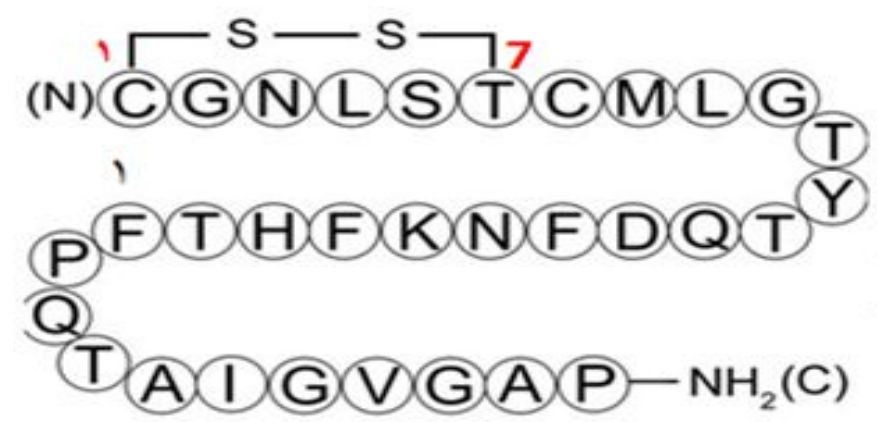

32

Figure S.1. Structure of calcitonin 
S2

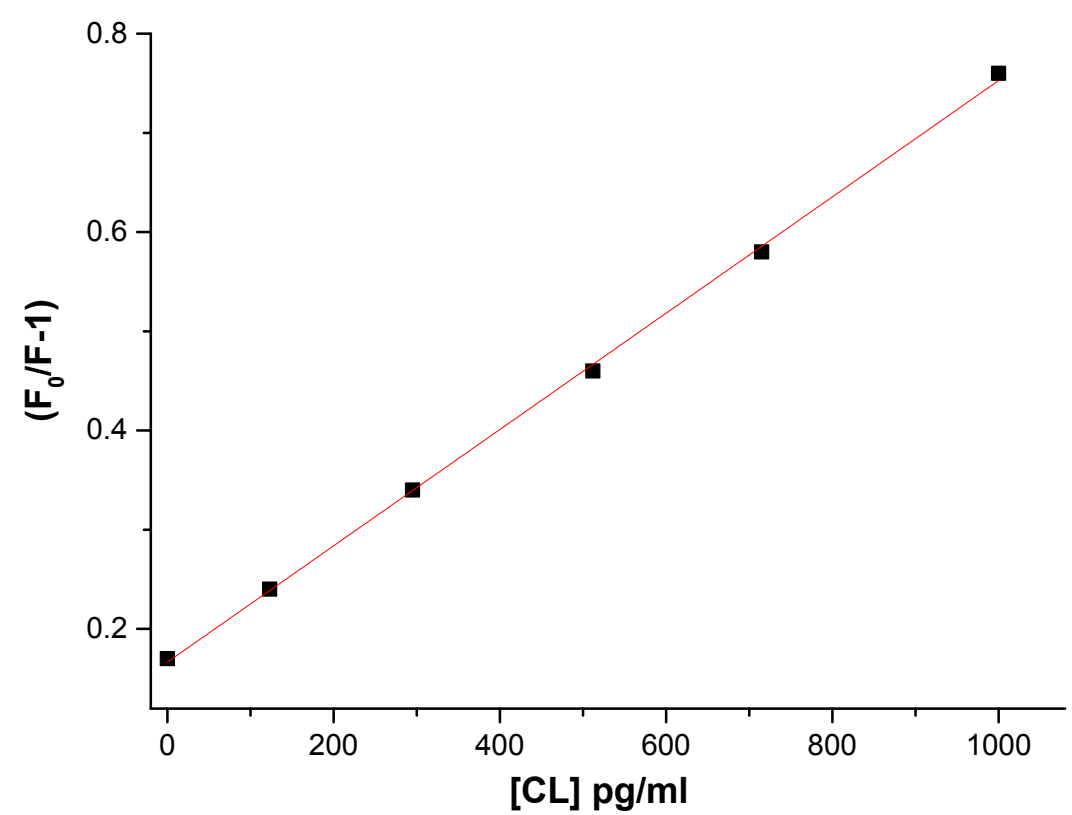

Figure S2. Stern-Völmer plot. 
S3

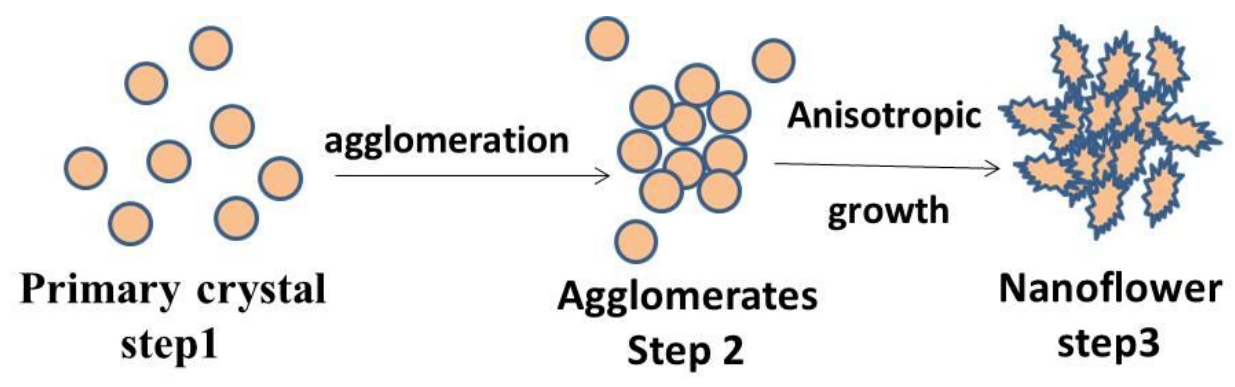

Figure S3. Mechanism of AuNFs formation in HEPES 


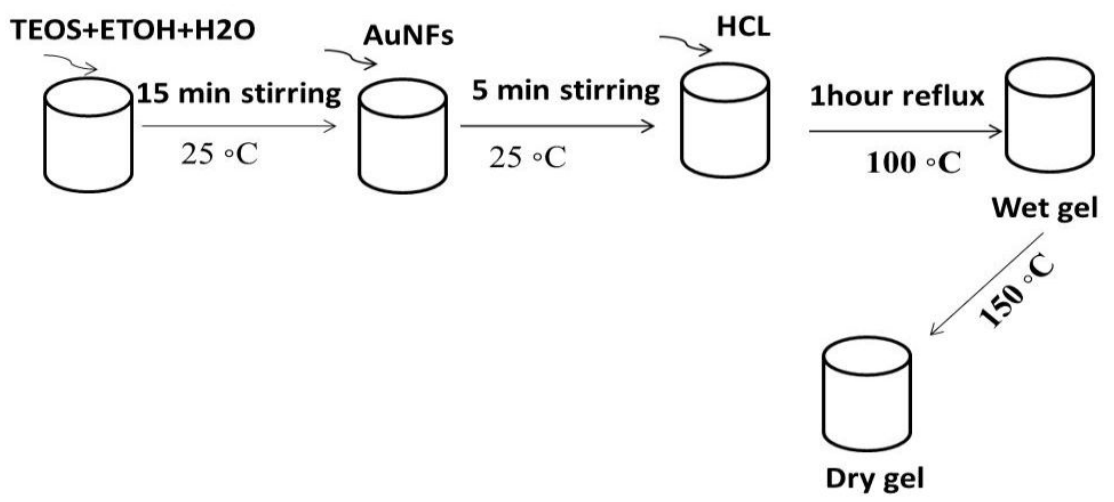

Figure S4. Mechanism of synthesis of Au doped in sol gel 
Table S1. Sensitivity and regression parameters for the proposed method

\begin{tabular}{ll}
\hline Parameter & Values \\
\hline$\lambda_{\text {em }}(\mathrm{nm})$ & 360 \\
Linear range, $\mathrm{pg} / \mathrm{mL}$ & $0.01-1000$ \\
Limit of detection (LOD), pg/mL & 0.707 \\
Limit of quantification (LOQ), pg/mL & 2.14 \\
Intercept (a) x $10^{-4}$ & 5.80 \\
Slope (b) & 0.16 \\
Standard deviation & 0.03 \\
Regression coefficient (r) & 0.99 \\
\end{tabular}

